\title{
Standardization of Diagnostics in Blood Safety
}

\author{
Jens Dreier ${ }^{a}$ Holger Hennig ${ }^{b}$ \\ ${ }^{a}$ Institute for Laboratory and Transfusion Medicine, Heart and Diabetes Center North Rhine Westphalia, Bad Oeynhausen, Germany; \\ ${ }^{b}$ Institute of Transfusion Medicine, University Hospital of Schleswig-Holstein, Lübeck, Germany
}

The implementation of nucleic acid amplification techniques (NAT) over the last two decades has totally revolutionized the field of transfusion medicine and blood safety. Several blood services started to screen for viral pathogens (HIV-1, HCV, HBV) with NAT on a voluntary basis in Germany in 1997. Later, the PaulEhrlich Institut (PEI) required that from April 1, 1999, NAT testing for HCV RNA is performed on all donations for the release of therapeutic blood components. The routine application of NAT required validation using appropriate standard reagents. In order to address this area scientifically, the WHO (World Health Organization) International Working Group on the Standardization of Gene Amplification Techniques (SoGAT) for the Virological Safety Testing of Blood and Blood Products was established at the National Institute for Biological Standards and Control (NIBSC), UK, in 1995. The purpose of this group is to promote the standardization of amplification methods through the exchange of information on scientific aspects of the technology, exchange of data on its evaluation for the testing of products, and the organization of international collaborative studies for the development, evaluation and provision of reference materials and working standards. Numerous in-house working reagents and reference panels existed in the pre-SoGAT era that used a variety of units, e.g. genome equivalents $/ \mathrm{ml}$, copies $/ \mathrm{ml}$ or PCR-detectable units $/ \mathrm{ml}$. The solution was to establish internationally accepted standards against which all working reagents could be calibrated [1-3].

Baylis et al. [4] give a fine overview on the history and actual developments of standardization of NAT for blood-borne pathogens in this issue of Transfusion Medicine and Hemotherapy. The current WHO International Standards (IS) for NAT and the International Reference Panels for different genotypes are described here in detail. The great advantage of the development of IS is the physically defined International Unit (IU), which allows the worldwide comparability of NAT test results and the replacement of standard preparations under maintenance of this measurement unit for the first time. Today, IS are widely used for the validation of newly developed NAT assays, e.g. by in vitro diagnostic (IVD) manufacturers, and for the calibration of secondary standards which are commonly used as run controls or in quality assurance proficiency panels.

It is difficult to cross-reference molecular assays or to validate novel NAT assays without well characterized reference materials. The lack of an IS for West Nile virus (WNV) RNA, for example, hindered the determination of the analytical sensitivity (95\% limit of detection). Therefore, the German authority PEI required the use of the Health Canada WNV Reference Reagent or similarly calibrated secondary standards for the quantification of assays. These standards are expressed in copies/ml and make comparison of results more difficult.

In addition to the standardization of NAT by the use of IS, the periodic review of the actual performance of methods is the second main pillar of quality assurance and quality control. Moreover, participation in external quality assurance programs is necessary. Several programs for proficiency testing for infectious diseases have been established in molecular diagnostics in Europe, including EQUALqual, a project proposed under the auspices of the European Communities Confederation of Clinical Chemistry and Laboratory Medicine (EC4) and funded by the European Commission, and several commercially available programs provided by, for example, Quality Control for Molecular Diagnostics (QCMD, UK), the United Kingdom National External Quality Assessment Service (NEQAS, UK), the Reference Institute for Bioanalytics (RfB, Germany), and the Institute for Standardization and Documentation in Medical Laboratory (INSTAND, Germany) [5]. Recently, a novel format for a personalized external quality assurance program (EQAP) was described that provides a collaborative trial for genomic detection of eight viruses simultaneously: HAV, HBV, HCV, HIV-1, HIV-2, parvovirus B19 (B19V), WNV and human cytomegalovirus (HCMV) [6]. The so-called MultiVir NAT is organized by the RfB, Germany. This EQAP enables flexible participation regarding the testing of 1-8 different transfusion-relevant viruses, qualitatively and/or quantitatively, and provides result reporting within 4 weeks. Barcoded samples can be processed as routine samples in an automated PCR workflow.

\section{KARGER}

Fax +497614520714 (c) 2015 S. Karger GmbH, Freiburg

$1660-3796 / 15 / 0424-0208 \$ 39.50 / 0$ 
Compared to viral pathogens, the transfusion-relevant bacteria have been neglected regarding quality assurance and quality control. Bacterial contamination of platelet concentrates (PC) remains a persistent problem in transfusion medicine. The residual risk of transfusion-related bacterial infections is currently 8- to 200 -fold higher than the residual risk of virus infections (e.g. HIV-1, HCV or HBV). Platelets are the main focus of bacterial contamination, due to the storage temperature of $22^{\circ} \mathrm{C}$ providing optimal growth conditions for a broad range of bacteria. Nevertheless, fatal septic reactions have also been reported after transfusion of contaminated red cell concentrates. The maximum shelf life for platelets was reduced from 5 to 4 days in Germany to increase blood safety. Alternatively, rapid screening methods for bacterial detection in platelets have been optimized over the last decade and are now available for routine screening [7].

The validation and head-to-head comparison of these rapid bacterial detection methods, however, represent a complicated process. An important criterion for the evaluation of an analytical method is the determination of the lower detection limit, requiring defined standards. However, standards are not yet available for microbiological diagnostics and are, thus, produced by each laboratory individually. Ascertaining the target values of these standards is extremely problematic because no metrologically correct measuring system for these parameters exists. Additionally, reference methods for bacterial quantification are not available. Target values for the so-called standards are, therefore, attained using either the routine methods biologically characterizing the standard or the same method used to screen the sample material. The routine methods used in microbiology are less reliable (incorrect and imprecise). The current practice is quantification via either i) bacterial titer expressed as colony-forming units ( $\mathrm{CFU}$ ) or ii) the nucleic acid molecule for detection expressed in copies or genome equivalents. Currently, defined, qualified microbiological standards are not yet available, with the exception of the WHO Repository of
Platelet Transfusion Relevant Bacteria Reference Strains, which were designed for the low-titer spiking of blood components with a guaranteed ability to multiply in PC. The International Society of Blood Transfusion (ISBT) Working Party Transfusion-Transmitted Infectious Diseases (WP-TTID), Subgroup Bacteria, organized an international study on bacteria standards to be used as a tool for development, validation, and comparison of both bacterial screening and pathogen reduction methods [8].

Vollmer et al. [9] present data in this issue on the first three individual collaborative trials for the detection of bacterial contamination in PC. The problems of standardization of bacterial detection methods with cultural and rapid methods were described in this study. Compared to viral parameters, an EQAP for the detection of bacterial contamination in PC has some special requirements: i) bacteria are supposed to have the ability to proliferate because some test principles are dependent on cell viability, but ii) titers have to be constant between sample setup and execution of analysis to obtain the highest reproducibility of results. This promising approach enables the head-to-head comparison of rapid and cultural bacterial detection systems for the first time. A special challenge is that the bacterial titer (expressed as CFU) is not static in samples or blood components, because microorganisms have the ability to proliferate during storage. By contrast, there is a direct correlation between viral load and copy number determined by NAT for viral approaches. For viral NAT assays, standardization was achieved by using well characterized reference material, i.e. calibrated material using a common standard unit (IU). For bacteria, there are several problems and questions regarding the establishment of a standard preparation. Bacteria are living organisms and, in contrast to viruses, are changeable regarding their metabolism.

Altogether, this issue of Transfusion Medicine AND HEMOTHERAPY provides an insight into the history and new developments of the standardization of diagnostics, and challenges and difficulties concerning the improvement of blood safety.

\section{References}

1 Robertson JS: International standardization of gene amplification technology. Biologicals 1998;26:111-113.

2 Saldanha J: Standardization: a progress report. Biologicals 1999;27:285-289.

3 Saldanha J: Validation and standardisation of nucleic acid amplification technology (NAT) assays for the detection of viral contamination of blood and blood products. J Clin Virol 2001;20:7-13.

4 Baylis S, Chudy M, Nübling M: Standardization of NAT for blood-borne pathogens. Transfus Med Hemother 2015;42: DOI: 10.1159/000435872.

5 Kessler HH, Raggam RB: Quality assurance and quality control in the routine molecular diagnostic laboratory for infectious diseases. Clin Chem Lab Med 2012;50: 1153-1159.
6 Schmidt M, Geilenkeuser WH, Kruse R: Proficiency panel with barcoded samples for eight transfusion relevant viruses improves external quality assurance to the next level. Transfus Med Hemother 2014;41(suppl 1):8.

7 Störmer M, Vollmer T: Diagnostic methods for platelet bacteria screening: current status and developments. Transfus Med Hemother 2014;41:19-27.

8 Störmer M, Arroyo A, Brachert J, Carrero H, Devine D, Epstein JS, Gabriel C, Gelber C, Goodrich R, Hanschmann KM, Heath DG, Jacobs MR, Keil S, de Korte D, Lambrecht B, Lee CK, Marcelis J, Marschner S, McDonald C, McGuane S, McKee M, Muller TH, Muthivhi T, Pettersson A, Radziwon P, Ramirez-Arcos S, Reesink HW, Rojo J, Rood I, Schmidt M, Schneider CK, Seifried E, Sicker U, Wendel S, Wood EM, Yomtovian RA, Montag T: Establishment of the first international repository for transfusion-relevant bacteria reference strains: ISBT Working Party TransfusionTransmitted Infectious Diseases (WP-TTID), Subgroup on Bacteria. Vox Sang 2012;102:22-31.
9 Vollmer T, Knabbe C, Geilenkeuser W-J, Schmidt M, Dreier J: Bench test for the detection of bacterial contamination in platelet concentrates using rapid and cultural detection methods with a standardized proficiency panel. Transfus Med Hemother 2015;42: DOI: $10.1159 / 000437396$. 